\title{
OPTIMAL SHAPE DESIGN USING DOMAIN TRANSFORMATIONS AND CONTINUOUS SENSITIVITY EQUATION METHODS
}

\author{
Lisa Stanley \\ Department of Mathematical Sciences \\ Montana State University \\ Bozeman, Montana * \\ stanley@math.montana.edu
}

\begin{abstract}
In this paper, we consider two approaches to solving an optimization based design problem where "shape" is the design parameter. Both methods use domain transformations to compute gradients. However, they differ in that the second method is based on solving a transformed optimization problem completely in the computational domain. We illustrate the methods using a simple 1D problem and discuss the benefits and drawbacks of each approach.
\end{abstract}

Keywords: Continuous Sensitivity Equation Methods, Optimal Design

\section{Introduction}

The focus of the paper is an optimal design problem where the design parameter determines the shape of the domain of the constraint equation. The cost function is given in terms of an integral expression describing the $L_{2}$ difference between some target function and the state variable. The constraint equation, or state equation, takes the form of an elliptic partial differential equation defined on a parameter dependent domain. Under the assumption that each point in the design space determines a unique state variable through the solution of the state equation, we pose the unconstrained optimal design problem.

\footnotetext{
*Work supported by the National Science Foundation under grant DMS-0072438 and the Air
} Force Office of Scientific Research under AASERT grant F49620-97-1-0329.

The original version of this chapter was revised: The copyright line was incorrect. This has been corrected. The Erratum to this chapter is available at DOI: 10.1007/978-0-387-35699-0_19 
Since the domain of the constraint equation changes with perturbations in the design, numerical solution of the optimal design problem is often hampered by burdensome grid generation requirements at each iteration of an optimization algorithm. One technique that can be used to avoid this problem is to transform the domain of the constraint equation to one that is fixed and no longer depends on the shape parameter. An equivalent transformed constraint equation is posed on this fixed, computational domain, see $[4,8]$, for example. In this paper, we present two approaches to the optimal design problem. Each approach uses the transformation technique mentioned above along with CSEMs (Continuous Sensitivity Equation Methods) in order to solve the optimal design problem. The main difference between the two methods is that one solves the optimal design problem using the parameter dependent domain of the constraint equation while the second approach applies a mapping technique in order to transform both the cost function and the constraint equation to a fixed computational space. This results in a transformed optimization problem. In each case, gradient based optimization is applied, and CSEMs are used to supply gradient information.

One of the major topics of concern for using CSEMs with optimal design is the issue of consistent derivatives. Within the optimization literature, the assumption is usually made that the gradient information is the derivative (with respect to the design parameter) of the numerical approximation of the cost function. There is a great deal of concern that convergence and robustness are compromised if the derivative approximations are computed using techniques which do not account for truncation and roundoff errors implicitly contained in the cost function. In $[1,2]$, the notion of asymptotically consistent derivatives is introduced, and CSEMs, when coupled with a trust region method, are shown to be applicable within optimal design algorithms. More precise definitions are introduced in Section 5.1. We first pose an example optimal design problem, and the computational approaches mentioned above are sketched out in the context of this example. Numerical results are shown, and we conclude with some general remarks concerning these approaches in Section 6.

\section{A 1D Optimal Design Problem}

Let $\mathcal{Q}=[1,+\infty)$ denote the design space, and for $q \in \mathcal{Q}$, let $\Omega_{q}=$ $(0, q)$. Consider the boundary value problem

$$
-\frac{d^{2}}{d x^{2}} \mathbf{w}(x ; q)=\mathbf{f}(x), \quad x \in \Omega_{q}
$$


with homogeneous Dirichlet boundary conditions

$$
\mathbf{w}(0)=0, \quad \mathbf{w}(q)=0 .
$$

The forcing function, $\mathbf{f}:(0,+\infty) \rightarrow \mathbb{R}$, is the piecewise continuous function defined by

$$
\mathbf{f}(x)=\left\{\begin{array}{cl}
0, & 0<x<1 \\
-1, & 1 \leq x<+\infty
\end{array}\right.
$$

For each $q \in \mathcal{Q},(1)-(2)$ has a unique solution $\mathbf{w}(\cdot ; q) \in H^{2}(0, q) \cap$ $H_{0}^{1}(0, q)$. Thus, we define a cost function $F: \mathcal{Q} \rightarrow \mathbb{R}$ by

$$
F(q)=\frac{1}{2} \int_{0}^{1}[\mathbf{w}(x ; q)+\sin (\pi x)]^{2} d x
$$

and we focus on the optimal design problem:

$$
\min _{q \in \mathcal{Q}} F(q) \text {. }
$$

Observe that the state equation, (1) - (2), is defined on the "physical" space $\Omega_{q}$, and the cost function, $F(\cdot)$, is defined over a fixed subset of this space. For this simple example, $q$ can be interpreted as a "shape" parameter in the sense that it determines the length of the interval over which the state $\mathbf{w}(\cdot ; q)$ is defined.

\subsection{Domain Transformations}

For large scale problems where the shape of the domain of the state equation is parameter dependent, grid generation often poses a major difficulty in the optimal design process. As mentioned earlier, one way to overcome this obstacle is to apply a domain transformation from the physical space to the fixed, computational space. For the model problem discussed in this paper, transforming is clearly very simple. We note that determining the domain transformation for any given two-dimensional or three-dimensional set can be much more complicated. Moreover, this calculation often requires the application of a numerical method. In order to focus on the issues related to sensitivity computation and the resulting gradient approximations, the application of an algebraic domain mapping to the model problem is justified.

Here we describe the transformation of the parameter dependent domain $[0, q]$ to the fixed computational domain, $[0,1]$. Once this mapping is constructed, the transformed state equation is defined accordingly. For $\alpha>0$, let $\Omega_{\alpha}=(0, \alpha)$, and for each fixed $q>1$, define the transformation $M(\cdot ; q): \bar{\Omega}_{1} \rightarrow \bar{\Omega}_{\alpha}$ by

$$
M(\xi, q)=\xi q=x
$$


Note that the spatial variable on the fixed domain is $\xi$, and we use $x$ to denote the spatial variable on $\Omega_{q}$. The transformations given above are used to define the "transformed" functions. Let $\xi \in \Omega_{1}$ and $q>1$, and for any function $\mathbf{u} \in H_{0}^{1}\left(\Omega_{q}\right)$, define the transformed function $\hat{\mathbf{u}} \in$ $H_{0}^{1}\left(\Omega_{1}\right)$ as follows

$$
\hat{\mathbf{u}}(\xi ; q)=\mathbf{u}(M(\xi, q) ; q)=\mathbf{u}(x ; q) .
$$

It can be shown that for a given value of $q$, if $\mathbf{w}(\cdot ; q)$ is a solution to the boundary value problem given in (1)-(2), then the corresponding function $\hat{\mathbf{w}}(\cdot ; q)$ is a solution to the boundary value problem

$$
-\frac{d^{2}}{d \xi^{2}} \hat{\mathbf{w}}(\xi)=q^{2} \hat{\mathbf{f}}(\xi, q), \quad \xi \in(0,1)
$$

with boundary conditions

$$
\hat{\mathbf{w}}(0)=0, \quad \hat{\mathbf{w}}(1)=0 .
$$

The forcing function $\hat{\mathbf{f}}(\xi ; q)$ is obtained by using the mapping $M$ and the relation $\hat{\mathbf{f}}(\xi ; q)=\mathbf{f}(M(\xi, q), q)=\mathbf{f}(x)$ and has the form

$$
\hat{\mathbf{f}}(\xi, q)= \begin{cases}0, & 0<\xi<\frac{1}{q} \\ -1, & \frac{1}{q} \leq \xi<1 .\end{cases}
$$

Henceforth, the boundary value problem (8)-(9) is referred to as the transformed state equation, and it is used in each of the computational approaches described in the following sections.

\section{Computational Approach 1}

In this section, we describe one approach for solving the optimal design problem in (5). This approach can be described as a "differentiate-thenmap" scheme. Observe that the gradient of the cost function has the form

$$
\nabla F(q)=\frac{d}{d q} F(q)=\int_{0}^{1}[\mathbf{w}(x ; q)+\sin (\pi x)] \mathbf{s}(x ; q) d x,
$$

where the sensitivity is defined as follows

$$
\mathbf{s}(\cdot ; q) \triangleq \frac{\partial}{\partial q} \mathbf{w}(\cdot ; q) .
$$

In order to compute the sensitivity, we use the CSEM approach. We derive a sensitivity equation, an equation for which the sensitivity in 
(12) is a solution. Formally speaking, this equation is derived by implicit "differentiation" of the state equation and boundary conditions in (1)(2). For the model problem considered here, it can be shown that the sensitivity equation and associated boundary conditions are given by

$$
-\frac{d^{2}}{d x^{2}} \mathbf{s}(x)=0, \quad x \in \Omega_{q}
$$

with boundary conditions

$$
\mathbf{s}(0)=0, \quad \mathbf{s}(q)=-\left.\frac{d}{d x} \mathbf{w}(x)\right|_{x=q}=-\frac{d}{d x} \mathbf{w}(q) .
$$

Observe that the normal derivative of $\mathbf{w}$ appears in the right boundary condition in (14). This is typical for shape sensitivity problems, and these boundary conditions are tricky to derive correctly for more complicated problems.

Gradient based optimization requires that we numerically approximate both the cost function and its gradient for a given value of the parameter $q$. Aside from the implementation of a quadrature rule, each iteration of the optimization algorithm involves a numerical calculation of both the state and the sensitivity for a given design parameter value. The following section describes the numerical scheme employed for these computations.

\subsection{State and Sensitivity Calculations}

Here we illustrate the use of the mapping technique discussed in Section 2.1. Both a transformed state equation and a transformed sensitivity equation are constructed on the computational domain $\Omega_{1}$. The derivation of the transformed state equation is presented in detail in Section 2.1 and is given explicitly in equations (8)-(9). In a similar fashion, we define the transformed sensitivity

$$
\hat{\mathbf{s}}(\xi ; q)=\mathbf{s}(M(\xi, q) ; q)=\mathbf{s}(x ; q),
$$

and the transformed sensitivity equation is constructed. This boundary value problem has the form

$$
-\hat{\mathbf{s}}^{\prime \prime}(\xi)=0, \quad \xi \in(0,1),
$$

with boundary conditions

$$
\hat{\mathbf{s}}(0)=0, \quad \hat{\mathbf{s}}(1)=-\left.\left(\frac{1}{q}\right) \cdot \frac{d}{d \xi} \hat{\mathbf{w}}(\xi)\right|_{\xi=1}=-\left(\frac{1}{q}\right) \cdot \frac{d}{d \xi} \hat{\mathbf{w}}(1) .
$$


Once the transformed equations are constructed, a discretization is applied. For the numerical approximations presented here, we apply a piecewise linear finite element method to (8)-(9) and to (16)-(17). For the sake of brevity, the details of the implementation are omitted; however, the interested reader can refer to [3] for a more complete exposition. Once the numerical calculations are performed, the recovery of the state and sensitivity approximations (defined on the physical space $\Omega_{q}$ ) is achieved through the relations in (7) and (15).

\section{Computational Approach 2}

In this section, we present an approach to the optimal design problem which is similar to an idea considered in [5]. Like the previous scheme, both domain transformations and CSEMs are used in this strategy. The fundamental difference between the following approach and the one presented in Section 3 is the order in which these techniques are applied. In this section, the domain transformation is applied to the cost function as well as the state equation. First, we construct a transformed optimal design problem which is equivalent to the original in (4) and which uses information from the transformed state equation. A CSEM is then used to supply gradient information for the transformed cost function.

Before presenting the transformed optimal design problem, we remark that under the mapping $M$ defined in (6), the following equality holds

$$
\int_{0}^{1} g(x) d x=\int_{M(0 ; q)}^{M(1 ; q)} g(M(\xi, q))\left|\frac{d M}{d \xi}\right| d \xi=q \int_{0}^{\frac{1}{q}} g(M(\xi, q)) d \xi
$$

where $g$ is any $C^{1}$-function defined on $\Omega_{1}$. Along with the previous equality, the definitions in (6) and (7) give rise to the transformed cost function

$$
\hat{F}(q)=\frac{1}{2} q \int_{0}^{\frac{1}{q}}[\hat{\mathbf{w}}(\xi, q)+\sin (q \pi \xi)]^{2} d \xi .
$$

Here $\hat{\mathbf{w}}(\xi, q)$ is the solution to the transformed state equation given by the boundary value problem (8)-(9) for each $q \in \mathcal{Q}$. Hence, the transformed optimal design problem is given by

$$
\min _{q \in \mathcal{Q}} \hat{F}(q)
$$

where the design space, $\mathcal{Q}$, remains the same as in Section 2. Observe that a factor of $q$ appears in the expression (18). Recall that the mapping, $M$, depends explicitly on the parameter $q$. Hence, the absolute value of the derivative of the mapping (and more generally, the determinant of the Jacobian matrix) is also parameter dependent, and this term 
appears explicitly in (18). For this particular example, the derivative is very simple, but we remark that the issue of parameter dependent derivatives is more complicated for two-dimensional and three-dimensional domains. A two-dimensional illustration can be found on pages 365-366 of [4].

Once the transformed optimal design problem is constructed, we proceed in much the same manner as previously discussed. Using Leibnitz' formula, the gradient of the transformed cost function has the following form

$$
\begin{aligned}
\nabla \hat{F}(q) & =\frac{1}{2} \int_{0}^{\frac{1}{q}}[\hat{\mathbf{w}}(\xi, q)+\sin (q \pi \xi)]^{2} d \xi+\left(\frac{q}{2}\right)\left[\hat{\mathbf{w}}\left(\frac{1}{q} ; q\right)\right]^{2}\left(\frac{-1}{q^{2}}\right) \\
& +\left(\frac{q}{2}\right) \int_{0}^{\frac{1}{q}} 2[\hat{\mathbf{w}}(\xi ; q)+\sin (q \pi \xi)]\left(\frac{\partial}{\partial q} \hat{\mathbf{w}}(\xi ; q)+\pi \xi \cos (q \pi \xi)\right) d \xi
\end{aligned}
$$

which can be simplified to the expression

$$
\begin{aligned}
\nabla \hat{F}(q)= & q \int_{0}^{\frac{1}{q}}[\hat{\mathbf{w}}(\xi, q)+\sin (q \pi \xi)][\mathbf{p}(\xi, q)+\pi \xi \cos (q \pi \xi)] d \xi \\
& +\frac{1}{2 q}\left(2 \hat{F}(q)-\left[\hat{\mathbf{w}}\left(\frac{1}{q} ; q\right)\right]^{2}\right) .
\end{aligned}
$$

In the equation above, the notation $\mathbf{p}(\xi ; q)$ is used to denote the sensitivity of the transformed state; that is, we define

$$
\mathbf{p}(\xi ; q) \triangleq \frac{\partial}{\partial q} \hat{\mathbf{w}}(\xi ; q) .
$$

It is important to note that the sensitivity of the transformed state, $\mathbf{p}(\xi ; q)$, is related to, but not the same function as, the transformed sensitivity, $\hat{\mathbf{s}}(\xi ; q)$. The notation used above reflects this important distinction. The following section describes the techniques used for obtaining numerical approximations for the transformed state and the sensitivity, $\mathbf{p}(\xi ; q)$.

\subsection{State and Sensitivity Calculations}

For this approach, the optimization algorithm requires that we compute a numerical approximation to the transformed state, $\hat{\mathbf{w}}(\cdot ; q)$, and the sensitivity of the transformed state, $\mathbf{p}(\cdot ; q)$. As in the previous section, a piecewise linear finite element method is used to approximate the transformed state $\hat{\mathbf{w}}(\xi, q)$.

In order to calculate an approximation for $\mathbf{p}(\cdot ; q)$, we derive a sensitivity equation for which $\mathbf{p}(\cdot ; q)$ is a solution. In particular, the transformed 
state equation in (8)-(9) is "differentiated". Although the parameter appears explicitly in the right hand side of equation (8) and determines a point of discontinuity of the forcing function, one can still derive the sensitivity equation in a mathematically precise fashion. A rigorous mathematical construction is presented in [6] and references therein. Here we simply state that the sensitivity, $\mathbf{p}(\cdot ; q)$, satisfies the second order, linear elliptic boundary value problem given by

$$
\begin{array}{cl}
-\frac{d^{2}}{d \xi^{2}} \mathbf{p}(\xi, q)= & 2 q \hat{\mathbf{f}}(\xi, q)-\delta_{\frac{1}{q}}(\xi), \\
\mathbf{p}(0)=0, & \mathbf{p}(1)=0 .
\end{array}
$$

Here $\delta_{\frac{1}{q}}(\xi)$ is the Dirac delta function with mass at $\xi=\frac{1}{q}$. Since the domain $\Omega_{1}$ does not depend on $q$, the boundary conditions are clear. Observe that the sensitivity equation is decoupled from the transformed state equation, but we caution the reader that this decoupling is merely a phenomena of the linearity of the transformed state equation. We also note that the linear elliptic problem (22)-(23) does not have a solution in $H_{0}^{2}\left(\Omega_{1}\right)$, and the system must be interpreted in the weak sense, that is, in integral form. For the results presented in this paper, a piecewise linear finite element method is used to approximate both $\hat{\mathbf{w}}(\cdot ; q)$ and $\mathbf{p}(\cdot ; q)$. For the sake of brevity, the details of the finite element implementations are omitted, and we proceed directly to the computational results.

\section{Computational Results}

In this section, numerical results are presented for two cases. The first is a comparison using a four-point Gauss quadrature rule for both the cost function approximations and the gradient approximations. From the second we make an interesting anecdotal comment concerning the importance of choosing a quadrature rule with the appropriate degree of accuracy.

Recall that each computational approach involves discretizing and numerically computing an approximation to the transformed state equation (8)-(9). The distinction between the calculations is the fact that Computational Approach 1 recovers an approximation to the original state through the mapping, $M$, and implements the quadrature rule in the physical space while Computational Approach 2 applies the quadrature rule in the computational space. Since $M$ is a straightforward algebraic manipulation which can be "hard-wired", there is no loss in accuracy for the state approximation during the recovery process of Computational Algorithm 1. We briefly note that a four-point quadrature rule is sufficient to obtain an extremely accurate approximation to the true cost 
function in each case. Figures 1 and 2 show the respective cost function approximations plotted against the graph of the true cost function. The step in the parameter is $\Delta q=0.1$ over the parameter range given, and the transformed state approximations are obtained using $N=3$ grid points for these graphs. We also note that the error (measured in the vector norm, $\|\cdot\|_{\infty}$ ) in the cost function approximations is on the order of $10^{-4}$ for each of the computational algorithms. Now we move to the more interesting issue of gradient approximations.

Figure 1. True Cost Function and Approximations for Computational Approach 1

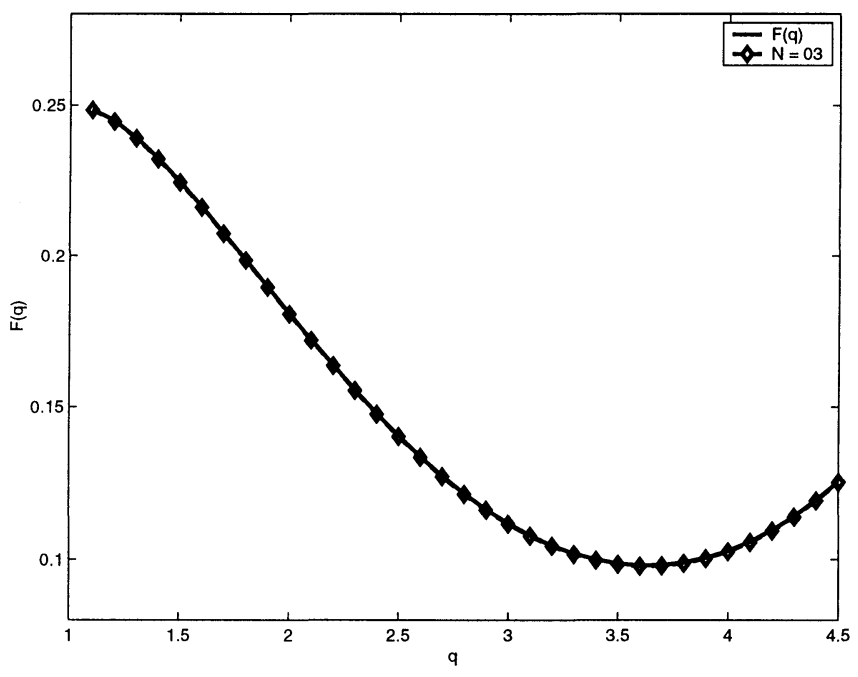

\subsection{Gradient Approximations}

This section briefly addresses the issue of gradient approximations for each computational approach. We preface the numerical results with two definitions regarding gradient approximations. The following discussion and definitions are taken from $[2,1]$. We remark that the notation in [1] is slightly different because they explore the issue of applying different approximation schemes to obtain the state and sensitivity approximations. For our results, the discretization applied to compute the state approximations, and subsequently the cost function approximations, is exactly the same as that applied to compute the sensitivity approximations and the subsequent gradient approximations. 
Figure 2. True Cost Function and Approximations for Computational Approach 2

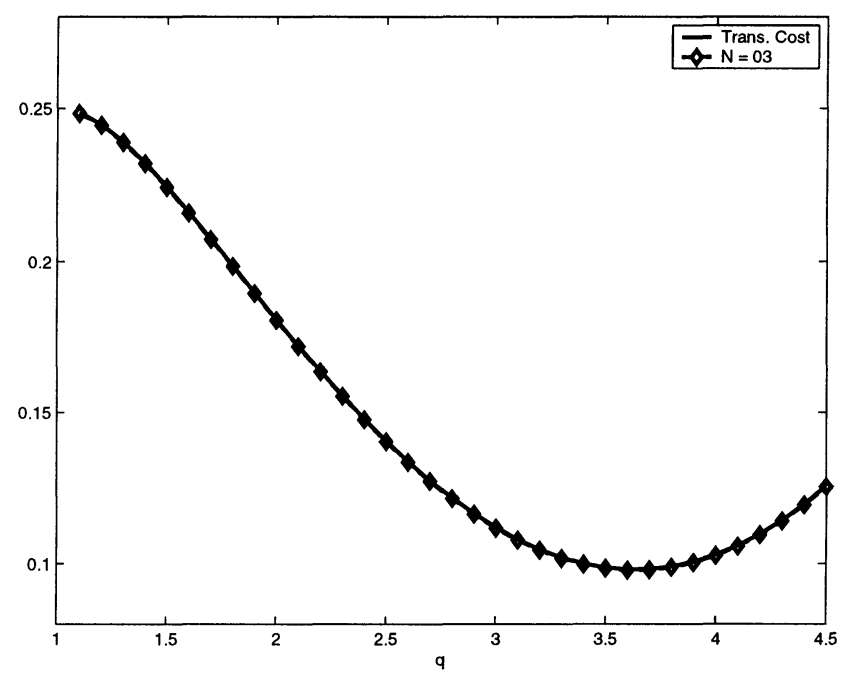

In the following discussion, we also refer to the discretization as an $N$-discretization in the sense that $N$ refers to the number of grid points in the finite element mesh. To be more precise, we should include notation identifying the quadrature rule here as well. However, since we are comparing approximations using a four point quadrature rule in both cases, we choose to simplify the notation as much as possible. Furthermore, $J$ denotes an arbitrary cost function which depends on the design parameter $q$. A sensitivity approach is said to produce consistent derivatives with respect to the state and sensitivity approximations using the $N$-discretization if

$$
\nabla J^{N}(q)=[\nabla J(q)]^{N} \quad \forall q \in Q .
$$

This definition states that the gradient of the approximate cost function is the same as the approximation of the true gradient. A more relaxed definition stipulates that the difference between the two gradient approximations should approach 0 with grid refinement. In particular, a sensitivity approach is said to produce asymptotically consistent derivatives with respect to the state and sensitivity approximations using the $N$-discretization if

$$
\left|\nabla J^{N}(q)-[\nabla J(q)]^{N}\right| \rightarrow 0 \quad \forall q \in Q
$$


Figure 3. True Gradient and Approximations for Computational Approach 1

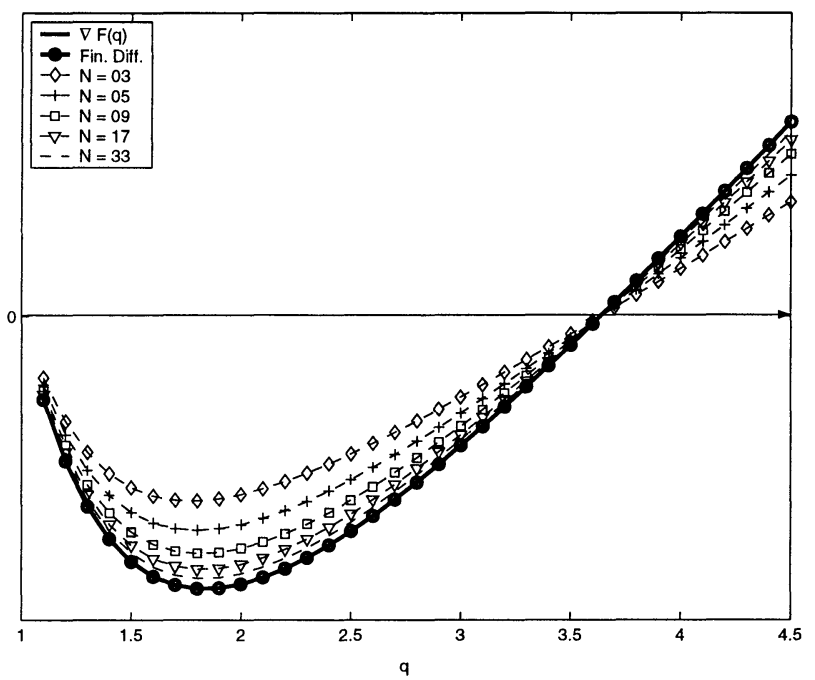

as $N \rightarrow \infty$, that is, as the grid is refined. The computational approaches presented in Sections 3 and 4 fall into the category of an approximation of the true gradient. Hence, Computational Approach 1 produces $[\nabla F(q)]^{N}$, and Computational Approach 2 yields $[\nabla \hat{F}(q)]^{N}$.

In the following figures, we present a sample of the gradient approximations obtained using each computational approach. The gradient approximations are compared with both a centered difference gradient approximation (solid curve with o's, representing $\nabla F^{N}$ and $\nabla \hat{F}^{N}$, respectively) and the true gradient (solid curve). In Figure 3, the gradient approximations generated using Computational Approach 1 converge to the finite difference gradient (and to the true gradient) with mesh refinement. Hence, Computational Approach 1 yields asymptotically consistent derivatives. Figure 4 indicates that Computational Approach 2 produces consistent derivatives.

\subsection{Anecdotal Observation}

In the case where a three-point Gauss quadrature rule is used, the quadrature rule is insufficient for convergence of the cost function approximations as the mesh is refined. That is, if we use three quadrature points for the integral approximations, then the cost function approxima- 
Figure 4. True Gradient and Approximations for Computational Approach 2

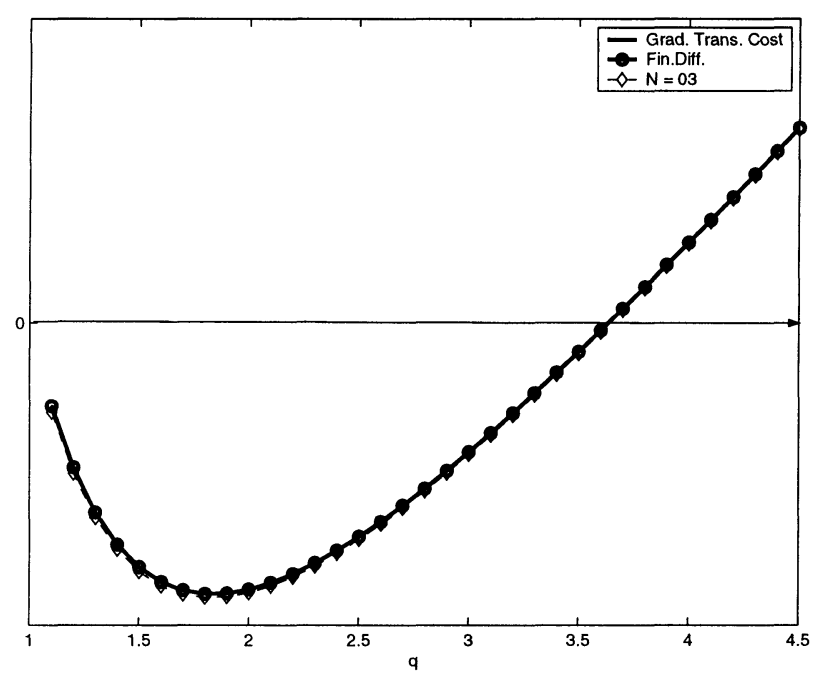

tions for each approach are given in Figures 5 and 6 . We have included the graphs for $N=3$ grid points and $N=33$ grid points to show that the accuracy of the approximations does not improve with mesh refinement, and the error (using $\|\cdot\|_{\infty}$ ) in these approximations is on the order of $10^{-3}$. All of the approximations generated using values of $N$ between 3 and 33 exhibit exactly the same behavior. The gradient approximations for this case are somewhat interesting. In particular, Figures 7 and 8 suggest that Computational Algorithm 1 produces asymptotically consistent gradients while Computational Approach 2 produces inconsistent or "non-consistent" gradient approximations. This behavior may be a result of the fact that the we use the transformed cost function approximation during the gradient calculation on the computational space, recall the expression in (20). The gradient expression for Computational Algorithm 1, in (11), does not explicitly involve the cost function, $F(q)$.

\section{Computational Issues}

We conclude with some observations gathered during the course of the research. Since the domain transformations depend explicitly on the parameter, spatial derivatives are also parameter dependent and appear ex- 
Figure 5. True Cost Function and Approximations for Computational Approach 1 using three-point quadrature rule

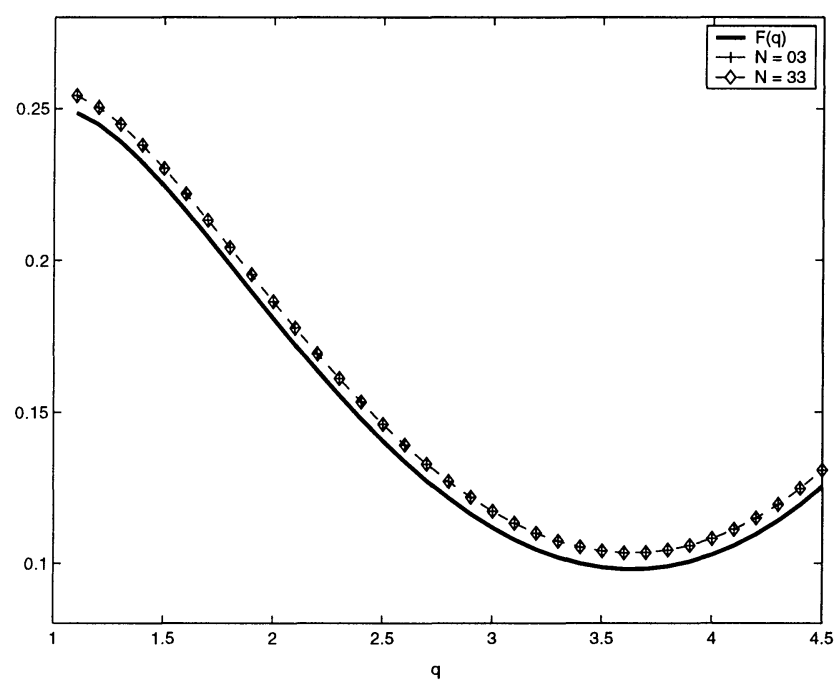

Figure 6. True Cost Function and Approximations for Computational Approach 2 using three-point quadrature rule

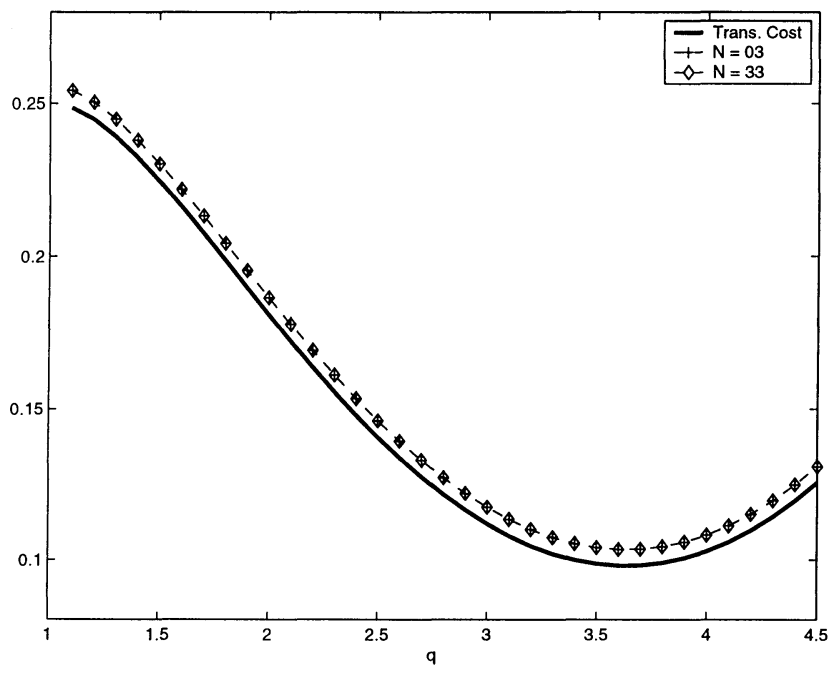


Figure 7. True gradient, finite difference gradient and approximations for Computational Approach 1 using three-point quadrature rule

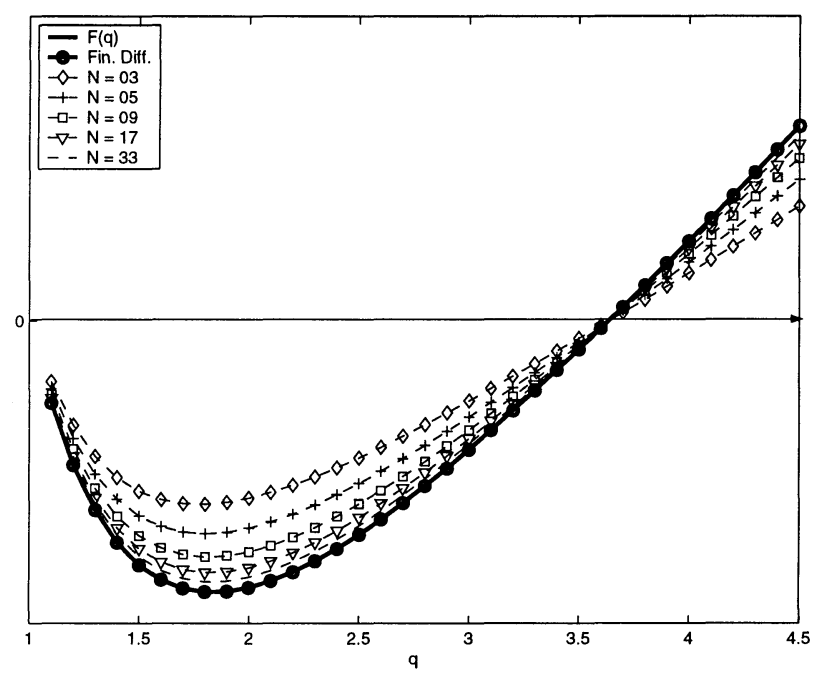

Figure 8. True gradient, finite difference gradient and approximations for Computational Approach 2 using three-point quadrature rule

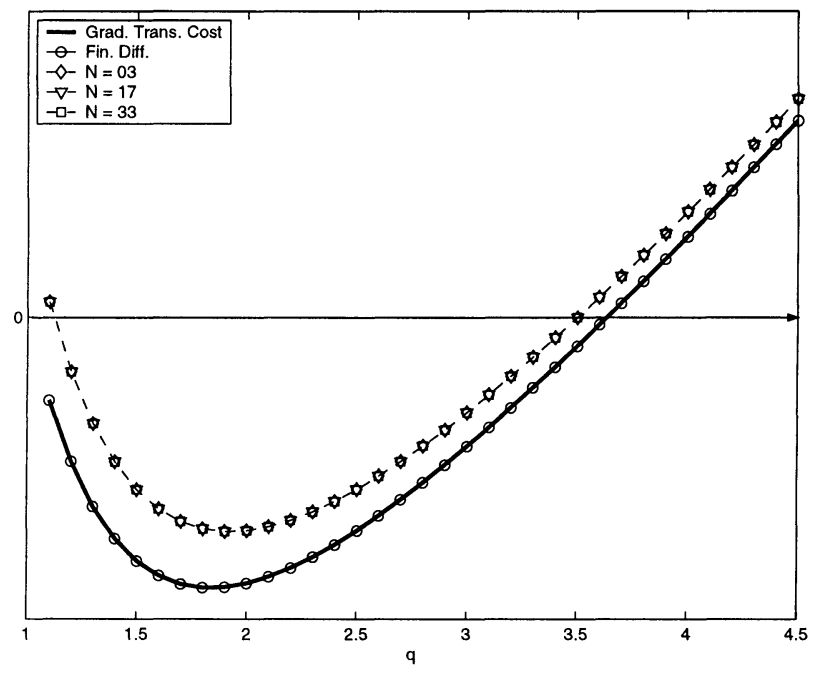


plicitly in both the transformed cost function and the transformed state equation. As a result, the derivation of a gradient expression is tedious and involves several terms including the transformed cost function, $\hat{F}(q)$. We approximate $\hat{F}(q)$ at each iteration of the optimization algorithm, and the approximation is reused in the gradient approximation routine. However, this may require good judgement for the quadrature rules as shown in Section 5.2. The results given here indicate that CSEMs can yield accurate, consistent gradients provided that the numerical schemes are chosen with care. Using the domain transformations is advantageous for the rigorous mathematical derivation of sensitivity equations. However, the issue of differentiability of the mappings becomes an important question for both gradient derivation and sensitivity analysis in Computational Approach 2 for problems with complicated geometries.

In Computational Approach 1, the derivation of the sensitivity equation is somewhat ad hoc; however, differentiation of the domain mapping is not required. One must also be willing to accept the asymptotically consistent derivatives that this method produces. For many problems, we observe that the gradient approximations for this approach tend to accurately pinpoint the location of the root of the gradient even on coarse meshes. Finally, the results given in Section 5.2 indicate that for certain problems, CSEMs can produce asymptotically consistent gradients even if the cost function approximations are inaccurate. Each computational algorithm exhibits specific characteristics that can be view as advantageous. Further research to determine which computational approach best fits a given problem is needed.

\section{Acknowledgments}

The author wishes to acknowledge Dr. John Burns and Dr. Eugene Cliff for raising questions which led to this research and Dr. Jeff Borggaard for many helpful conversations concerning optimization.

\section{References}

[1] J.T. Borggaard and J.A. Burns. (1997). Asymptotically Consistent Gradients in Optimal Design. Multidisciplinary design optimization, State of the Art, 303314, 1997.

[2] J.T. Borggaard (1994). The Sensitivity Equation Method for Optimal Design. $\mathrm{PhD}$ thesis, Virginia Polytechnic Institute and State University, Blacksburg, Virginia. Mathematics PhD.

[3] J.A. Burns and L. G. Stanley. (2001). A Note on the Use of Transformations in Sensitivity Computations for Elliptic Systems. Mathematical and Computer Modelling, 33, 101-114. 
[4] K.A. Hoffman and S.T. Chiang. (1993). Computational Fluid Dynamics for Engineers. Engineering Education System.

[5] M. Laumen. (2000). Newton's Method for a Class of Optimal Shape Design Problems. SIAM Journal on Optimization, 10(2), 503-533.

[6] L.G. Stanley. (2001) Sensitivity Equation Methods for Parameter Dependent Elliptic Equations. Numerical Functional Analysis and Optimization, 10(5\&6), 721-748.

[7] L.G. Stanley. (2001). Shape Sensitivities for Optimal Design: A Case Study on the Use of Continuous Sensitivity Equation Methods. David Gao, Ray Ogden and Georgios Stavroulakis, editors, Nonsmooth / Nonconvex Mechanics: Modeling, Analysis and Numerical Methods, pages 369-384. Kluwer Academic Publishers, Nonconvex Optimization and Its Applications Series (50), The Netherlands, 2001.

[8] J.F. Thompson and Z.U. Warsi and C.W. Mastin. (1985). Numerical Grid Generation Foundations and Applications. Elsevier Publishing Company, 1985. 